\title{
A Novel Reliability Evaluation Method Based on RBD and AHP for Industrial Network Systems
}

\author{
Yongni $\mathrm{Li}^{1, \mathrm{a}}$, Zhenxing $\mathrm{Li}^{1}$, Baiyi $\mathrm{Li}^{1}$ and Song $\mathrm{Hu}^{1}$ \\ ${ }^{1}$ CRRC QINGDAO SIFANG CO., LTD., Shandong Qingdao 266111, China
}

\begin{abstract}
Since each component has different impacts on reliability of the industrial network system, a multi-layer reliability evaluation method was proposed in this paper. Firstly, in order to construct multi-layer reliability evaluation system, a framework of industrial network system was introduced based on analytic hierarchy process (AHP). Secondly, a multi-layer reliability evaluation model with weight coefficient of components was proposed based on reliability block diagram (RBD). Thirdly, the simple rule-based fuzzy judgment and risk priority number (RPN) were applied to determining weight coefficient. Last, a reliability evaluation case of the industrial network system for an electronic automatic assembly line was studied. It shows that the proposed method is more reasonable than the conventional reliability analysis method, and the reliability prediction result is consistent with the engineering practice.
\end{abstract}

\section{Introduction}

Reliability has become a common problem of industrial network system, and its indicator is considered as an important quality attribute, which has the same important status as technical and economic indicators such as system performance and cost. With the development of reliability engineering, the idea that reliability of product is the result of design, manufacture and management has been understood and accepted gradually. According to statistics, around $80 \%$ of the product reliability problems derive from the design stage of product, which means the design level directly influences the product reliability in practice. So, safety, reliability and efficiency of industrial network system depend on system reliability analysis, assessment, prediction and optimization during design phase.

As an important indicator of the product, reliability has got more and more attention. However, it is still difficult to evaluate the reliability of industrial network system comprehensively during design phase. Over the years, scholars all over the world have carried out a lot of fruitful research results on the problem. A series of reliability analysis methods have been put forward such as reliability block diagram(RBD)[1], binary decision diagram[2], fault tree(FT)[3], bayesian networks(BN)[4] and petri net[5,6]. As a simple, direct and efficient method, RBD has been widely applied to engineering. For example, Dr. Dou proposed reliability evaluation model of ship to air missile launching system on the basis of RBD, achieved the reliability evaluation assessment when the single shot happened[7]. Dr. Chen established reliability modeling process based on ExtendSim on the basis of RBD to solve the modeling and programming difficulties of traditional reliability method[8]. Dr. Wu studied the reliability of spacecraft solar wing system following RBD and FT[9]. Dr. Sun introduced a layered hybrid model method to analyse the reliability of the airplane control system which is based on combining RBD and generalized stochastic Petri net[10].

Though RBD can effectively build reliability model and evaluate reliability of the system, it ignores the fact that the industrial network system is a typical hybrid system integrated with parts, electrical equipment, electronic components, control components and other units, whose impacts on system reliability are different. For example, it is obvious that a server failure has a greater influence than a circuit failure on system reliability. Therefore, considering the different influence of units on system reliability is more reasonable in the construction of reliability modeling. Thereby, engineers can conveniently recognize the weak link and optimize the system reliability during design phase.

Based on the above consideration, a multi-layer reliability evaluation system was established by AHP method for the industrial network system. Then, a multilayer reliability evaluation model with weight coefficient of units was proposed. A case proved the effectiveness of the multi-layer reliability evaluation method which meets the needs of reliability evaluation in the development process of industrial network system.

\section{Overview of industrial network system}

Industrial network system is a full digital, bidirectional, multi-station communication system installed in the

\footnotetext{
a Corresponding author: 15151853987@163.com
} 
industrial production. It can be described that the system consists of a series of different hardware and software modules that play functions of data acquisition, data transmission, data processing and so on [11].

Industrial network system is a typical machine, electricity and control system. Any fault will result in production disruptions, even safety accidents, which will cause significant economic losses to the enterprise. As industrial 4.0 and made in China 2025 are put forward, quantities of industrial network system are rapidly increasing. Accordingly, reliability problems of industrial network system are constantly exposed and increasingly prominent. Due to the variety of parts, complex structure, high integration and so on, it is very difficult to accurately calculate the reliability of industrial network system by using the traditional reliability method. So, establishing a new reliability evaluation method that adapts to industrial network system is of great importance to improve reliability, reduce safety accidents and increase corporate profits.

\section{The multi-layer reliability model}

AHP is to decompose complex engineering problems from top to bottom to multiple levels and then solve the priority of each level to the upper level, so as to qualitatively and quantitatively analyze the problem or target. In this paper, system reliability model which reflects the importance of different units is constructed on the basis of AHP and RBD.

According to AHP method, industrial network system can be divided into four basic levels, namely system layer, subsystem layer, function module layer and basic module layer, as shown in figure 1. The upper layer is the target layer of next layer on reliability and the next layer is the basic unit of upper layer. The basic 4 layer can be increased or reduced in order to meet the analytic need for industrial network system with different complexity.

Every level reliability model is constructed based on RBD. For the series RBD, the reliability can be calculated as follows:

$$
R_{S}(t)=\prod_{i} R_{i}^{w_{i}}(t)
$$

where, $R_{S}$ represents target reliability of the layer, $t$ represents time, $R_{i}$ represents unit $i$ reliability of the layer, $w_{i}$ represents the weight of unit $i$.

For parallel RBD, the reliability can be calculated as follows:

$$
R_{S}(t)=1-\prod_{i}\left(1-R_{i}^{w_{i}}(t)\right)
$$

For next league or redundancy structure, reliability can be calculated by considering the impact of the weight coefficient. In theory, the weight coefficient $w_{i}$ belongs to $[0, \infty)$ in the above formula.

When $w_{i}$ equals to $0, R_{i}$ equals to 1 which shows that unit $i$ has no effect on reliability for target layer. When $w_{i}$ is close to $\infty, R_{i}$ is close to 0 which means that unit $i$ has a deterministic effect on reliability for target layer.

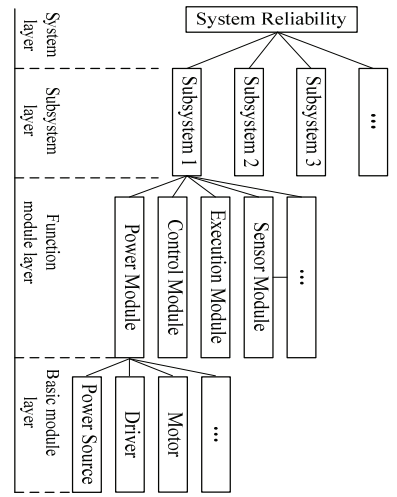

Figure 1. The multi-layer reliability evaluation system for industrial network system.

\section{Weight coefficient calculating method}

Weight is the most critical and difficult point when AHP method is used to analyze system reliability, that is to determine the weight coefficient in formula (1) and formula (2). AHP method needs to gain judgment matrix which can represent the relative importance of different index by experts[12]. Judgment matrix often adopts the proportional scaling method, and different proportion scales usually cause different results. Therefore, the fuzzy judgement matrix method is proposed in order to correctly evaluate system reliability by AHP in this paper. By the way, weight coefficients are able to truly reflect the relative importance of different index.

For the basic unit of each layer, index set needed to compare importance is as follows:

$$
R P N=\left\{R P N_{1}, R P N_{2}, \ldots, R P N_{n}\right\}
$$

where, $R P N_{i}$ is the risk priority number of unit $i$, $i=1,2, \ldots, n, n$ is total unit number. This paper calculates $R P N_{i}$ by combining the risk priority number method of Criticality Analysis (CA) with the failure model frequency rate[13]. The formula is as follows:

$$
R P N_{i}=\sum_{j} \alpha_{i j} \cdot O P R_{i j} \cdot E S R_{i j}
$$

where, $O P R$ is probability level of the failure model of the unit; ESR is the effect severe degree of the unit to target layer. $\alpha$ is failure model frequency rate which means ratio of the failure mode in all failure for the unit; $j=1,2, \cdots$ is failure model frequency number for the unit. The grading criteria of $O P R$ and ESR are presented in table 1 and table 2 , respectively.

Table 1. The grading criterion of $O P R$

\begin{tabular}{|c|c|c|}
\hline $\begin{array}{c}\text { OPR grading } \\
\text { level }\end{array}$ & $\begin{array}{c}\text { Probability } \\
\text { level }\end{array}$ & $\begin{array}{c}\text { Probability } \\
\text { range }\end{array}$ \\
\hline 1 & the lowest & $P \leq 10^{-6}$ \\
\hline 2,3 & lower & $10^{-6}<P \leq 10^{-4}$ \\
\hline $4,5,6$ & common & $10^{-4}<P \leq 10^{-2}$ \\
\hline 7,8 & higher & $10^{-2}<P \leq 10^{-1}$ \\
\hline 9,10 & the highest & $P>10^{-1}$ \\
\hline
\end{tabular}


Table 2. The grading criterion of ESR.

\begin{tabular}{|c|l|}
\hline $\begin{array}{c}\text { ESR grading } \\
\text { level }\end{array}$ & \multicolumn{1}{c|}{ ESR level } \\
\hline $1,2,3$ & $\begin{array}{l}\text { Mild (Do not lead to people injuries, } \\
\text { mild product damage, property loss } \\
\text { and environmental pollution, but } \\
\text { plan disorder) }\end{array}$ \\
\hline $4,5,6$ & $\begin{array}{l}\text { Medium (Lead to medium people } \\
\text { injuries, product damage, task delay } \\
\text { or demotion, medium property loss } \\
\text { and environmental pollution) }\end{array}$ \\
\hline 7,8 & $\begin{array}{l}\text { Deadly (Lead to severe people } \\
\text { injuries, product damage, task } \\
\text { failure, severe property loss and } \\
\text { environmental pollution) }\end{array}$ \\
\hline 9,10 & $\begin{array}{l}\text { Disastrous (Lead to the deaths of } \\
\text { personnel, product damage, major } \\
\text { property loss and disastrous } \\
\text { environmental pollution) }\end{array}$ \\
\hline
\end{tabular}

According to the result calculated by formula (4), $R P N_{i}$ and $R P N_{k}$ in index set $R P N$ takes binary comparison. If $R P N_{i}>R P N_{k}$, let $e_{i k}=0, e_{k i}=0$; If $R P N_{i}=R P N_{k}$, let $e_{i k}=0, e_{k i}=0$; If $R P N_{i}<R P N_{k}$, let $e_{i k}=0, e_{k i}=1$. The binary contrast matrix on index importance is as follows:

$$
E=\left[\begin{array}{cccc}
e_{11} & e_{12} & \cdots & e_{1 n} \\
e_{21} & e_{22} & \cdots & e_{2 n} \\
\cdots & \cdots & \cdots & \cdots \\
e_{n 1} & e_{n 2} & \cdots & e_{n n}
\end{array}\right]=\left(e_{i k}\right)
$$

Further, $f_{i}$ (the importance ranking index of $R P N_{i}$ ) can be calculated as follows:

$$
f_{i}=\sum_{k} e_{i k}
$$

Ranking $f_{i}$ by value, importance degree of reliability to upper layer is get accordingly for each unit. Thus, fuzzy judgement matrix $W$ is able to be calculated indirectly which is similar to the binary contrast matrix, as follows:

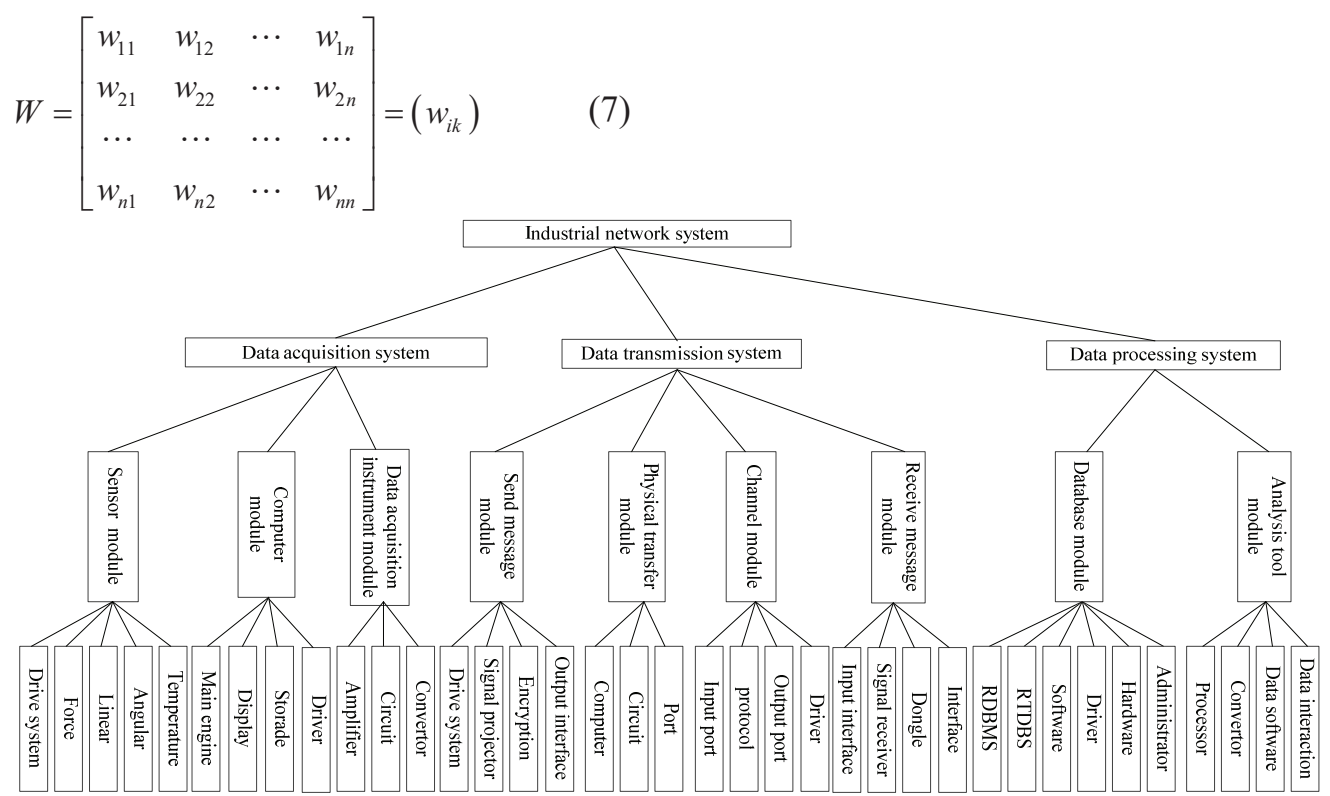

Figure 2. The multi-layer reliability evaluation system for the industrial network system of an electronic automatic assembly line. where,

$$
w_{i k}=\frac{f_{i}-f_{k}}{2(\mathrm{n}-1)}+0.5 \quad(i, k=1,2, \ldots, \mathrm{n})
$$

Through this transformation, the sort index of the index is used to transform the fuzzy indirect scale which can reflect the relative importance of each index. Construct a fuzzy judgment matrix $\left(w_{i k}\right)$, we can get the fuzzy membership degree per line. According to the nature of the fuzzy judgement matrix $W$, fuzzy membership of each row is able to be calculated as follows:

$$
w_{i}=\sum_{k} w_{i k} \quad(i \neq k)
$$

where, $w_{i}$ is the importance of index $i$ to the upper layer, namely weight coefficient.

\section{Case study}

The reliability of the industrial network system for an electronic automatic assembly line was studied by the proposed multi-layer reliability evaluation method. The industrial network system of the electronic automatic assembly line is composed of data acquisition system, data transmission system and data processing system, as shown in figure 2.

\subsection{Reliability analysis of module layer}

In the case, reliability of each component is assumed to be exponential distribution and failure rate is $\lambda$. Each component parameters of failure rate, $O P R$ and $E S R$ are show in table 3. Failure model number of each component does not be considered to simplify the calculation. According to formula (4) and (5), the binary contrast matrix of each system in function module layer is calculated as follows:

Table 3. The failure rate, $O P R$ and $E S R$ of components. 


\begin{tabular}{|c|c|c|c|c|c|c|c|}
\hline Name of parts & $\lambda\left(\mathbf{1 0}^{-6}\right)$ & $\boldsymbol{O P R}$ & $\boldsymbol{E S R}$ & Name of parts & $\lambda\left(\mathbf{1 0}^{-\mathbf{6}}\right)$ & $\boldsymbol{O P R}$ & $\boldsymbol{E S R}$ \\
\hline Drive system & 0.5 & 1 & 4 & Output interface & 1.5 & 2 & 1 \\
\hline Force & 15 & 2 & 3 & Computer & 0.5 & 1 & 3 \\
\hline Linear & 20 & 3 & 3 & Port & 2.5 & 1 & 4 \\
\hline Angular & 1.5 & 1 & 3 & Input port & 15.5 & 3 & 1 \\
\hline Temperature & 50.5 & 3 & 2 & Protocol & 18.5 & 2 & 4 \\
\hline Main engine & 15.5 & 2 & 3 & Output port & 110.5 & 4 & 4 \\
\hline Display & 130.5 & 4 & 1 & Input interface & 0.5 & 1 & 1 \\
\hline Storage & 12.5 & 2 & 2 & Signal receiver & 0.5 & 1 & 1 \\
\hline Driver & 1.5 & 2 & 1 & Dongle & 5.5 & 2 & 2 \\
\hline Amplifier & 0.5 & 1 & 4 & Interface & 8.5 & 2 & 1 \\
\hline Circuit & 3.5 & 2 & 3 & RDBMS & 5.5 & 2 & 4 \\
\hline Convertor & 55.5 & 3 & 1 & RTDBS & 0.5 & 1 & 4 \\
\hline Signal projector & 110.5 & 4 & 4 & Software & 23.5 & 3 & 3 \\
\hline Encryption & 185.5 & 4 & 2 & Hardware & 14.5 & 3 & 1 \\
\hline Administrator & 8.5 & 2 & 1 & Processor & 15.5 & 2 & 2 \\
\hline Data software & 80.5 & 3 & 4 & Data interaction & 0.5 & 1 & 3 \\
\hline
\end{tabular}

1) The binary contrast matrix of sensor module

$$
E=\left[\begin{array}{lllll}
0 & 0 & 0 & 1 & 0 \\
1 & 0 & 0 & 1 & 0 \\
1 & 1 & 0 & 1 & 1 \\
0 & 0 & 0 & 0 & 0 \\
1 & 0 & 0 & 1 & 0
\end{array}\right]
$$

2) The binary contrast matrix of computer module

$$
E=\left[\begin{array}{llll}
0 & 1 & 1 & 1 \\
0 & 0 & 0 & 1 \\
0 & 0 & 0 & 1 \\
0 & 0 & 0 & 0
\end{array}\right]
$$

3) The binary contrast matrix of data acquisition instrument module

$$
E=\left[\begin{array}{lll}
0 & 0 & 1 \\
1 & 0 & 1 \\
0 & 0 & 0
\end{array}\right]
$$

4) The binary contrast matrix of send message module

$$
E=\left[\begin{array}{llll}
0 & 0 & 0 & 1 \\
1 & 0 & 1 & 1 \\
1 & 0 & 0 & 1 \\
0 & 0 & 0 & 0
\end{array}\right]
$$

5) The binary contrast matrix of physical transfer module

$$
E=\left[\begin{array}{lll}
0 & 0 & 0 \\
1 & 0 & 1 \\
1 & 0 & 0
\end{array}\right]
$$

6) The binary contrast matrix of channel module

$$
E=\left[\begin{array}{llll}
0 & 0 & 0 & 1 \\
1 & 0 & 0 & 1 \\
1 & 1 & 0 & 1 \\
0 & 0 & 0 & 0
\end{array}\right]
$$

7) The binary contrast matrix of receive message module

$$
E=\left[\begin{array}{llll}
0 & 0 & 0 & 0 \\
0 & 0 & 0 & 0 \\
1 & 1 & 0 & 1 \\
1 & 1 & 0 & 0
\end{array}\right]
$$

8) The binary contrast matrix of database module

$$
E=\left[\begin{array}{llllll}
0 & 1 & 0 & 1 & 1 & 1 \\
0 & 0 & 0 & 1 & 1 & 1 \\
1 & 1 & 0 & 1 & 1 & 1 \\
0 & 0 & 0 & 0 & 0 & 0 \\
0 & 0 & 0 & 1 & 0 & 1 \\
0 & 0 & 0 & 0 & 0 & 0
\end{array}\right]
$$

9) The binary contrast matrix of analysis tool module

$$
E=\left[\begin{array}{llll}
0 & 1 & 0 & 1 \\
0 & 0 & 0 & 0 \\
1 & 1 & 0 & 1 \\
0 & 0 & 0 & 0
\end{array}\right]
$$

According to formula (6) (8), fuzzy judgment matrix of each function module can be obtained in turn as follows:

1) Fuzzy judgment matrix of sensor module

$$
W=\left[\begin{array}{ccccc}
0.5 & 3 / 8 & 1 / 8 & 5 / 8 & 3 / 8 \\
5 / 8 & 0.5 & 0.25 & 0.75 & 0.5 \\
7 / 8 & 0.75 & 0.5 & 1 & 0.75 \\
3 / 8 & 0.25 & 0 & 0.5 & 0.25 \\
5 / 8 & 0.5 & 0.25 & 0.75 & 0.5
\end{array}\right]
$$

2) Fuzzy judgment matrix of computer module

$$
W=\left[\begin{array}{cccc}
0.5 & 5 / 6 & 5 / 6 & 1 \\
1 / 6 & 0.5 & 0.5 & 2 / 3 \\
1 / 6 & 0.5 & 0.5 & 2 / 3 \\
0 & 1 / 3 & 1 / 3 & 0.5
\end{array}\right]
$$

3) Fuzzy judgment matrix of data acquisition instrument module

$$
W=\left[\begin{array}{ccc}
0.5 & 0.25 & 0.75 \\
0.75 & 0.5 & 1 \\
0.25 & 0 & 0.5
\end{array}\right]
$$

4) Fuzzy judgment matrix of send message module

$$
W=\left[\begin{array}{cccc}
0.5 & 1 / 6 & 1 / 3 & 2 / 3 \\
5 / 6 & 0.5 & 2 / 3 & 1 \\
2 / 3 & 1 / 3 & 0.5 & 5 / 6 \\
1 / 3 & 0 & 1 / 6 & 0.5
\end{array}\right]
$$

5) Fuzzy judgment matrix of physical transfer module

$$
W=\left[\begin{array}{ccc}
0.5 & 0 & 0.25 \\
1 & 0.5 & 0.75 \\
0.75 & 0.25 & 0.5
\end{array}\right]
$$

6) Fuzzy judgment matrix of channel module 


$$
W=\left[\begin{array}{cccc}
0.5 & 1 / 3 & 1 / 6 & 2 / 3 \\
2 / 3 & 0.5 & 1 / 3 & 5 / 6 \\
5 / 6 & 2 / 3 & 0.5 & 1 \\
1 / 3 & 1 / 6 & 0 & 0.5
\end{array}\right]
$$

7) Fuzzy judgment matrix of receive message module

$$
W=\left[\begin{array}{cccc}
0.5 & 0.5 & 0 & 1 / 6 \\
0.5 & 0.5 & 0 & 1 / 6 \\
1 & 1 & 0.5 & 2 / 3 \\
5 / 6 & 5 / 6 & 1 / 3 & 0.5
\end{array}\right]
$$

8) Fuzzy judgment matrix of database module

$$
W=\left[\begin{array}{cccccc}
0.5 & 0.6 & 0.4 & 0.9 & 0.7 & 0.9 \\
0.4 & 0.5 & 0.3 & 0.8 & 0.6 & 0.8 \\
0.6 & 0.7 & 0.5 & 1 & 0.8 & 1 \\
0.1 & 0.2 & 0 & 0.5 & 0.3 & 0.5 \\
0.3 & 0.4 & 0.2 & 0.7 & 0.5 & 0.7 \\
0.1 & 0.2 & 0 & 0.5 & 0.3 & 0.5
\end{array}\right]
$$

9) Fuzzy judgment matrix of analysis tool module

$$
W=\left[\begin{array}{cccc}
0.5 & 5 / 6 & 1 / 3 & 5 / 6 \\
1 / 6 & 0.5 & 0 & 0.5 \\
2 / 3 & 1 & 0.5 & 1 \\
1 / 6 & 0.5 & 0 & 0.5
\end{array}\right]
$$

According to formula (9) and above fuzzy judgment matrix, the weight coefficient of each component is evaluated. Then, reliability of each module which is shown in table 4 is obtained on the basis of series RBD when system continuously works for 1000 hours. The failure rate of each module is shown in table 5.

Table 4. Reliability of each function module.

\begin{tabular}{|c|c|}
\hline Module name & Reliability \\
\hline Sensor module & 0.811 \\
\hline Computer module & 0.792 \\
\hline $\begin{array}{c}\text { Data acquisition } \\
\text { instrument module }\end{array}$ & 0.979 \\
\hline Send message module & 0.539 \\
\hline Physical transfer module & 0.991 \\
\hline Channel module & 0.803 \\
\hline Receive message module & 0.969 \\
\hline Database module & 0.851 \\
\hline Analysis tool module & 0.754 \\
\hline
\end{tabular}

\subsection{Reliability analysis of subsystem}

$O P R$ and $E S R$ of each function module are shown in table 5.

Table 5. Failure rate, $O P R$ and $E S R$ of each function module.

\begin{tabular}{|c|c|c|c|}
\hline Module name & $\lambda\left(\mathbf{1 0}^{-4}\right)$ & $\begin{array}{c}\boldsymbol{O P} \\
\boldsymbol{R}\end{array}$ & $\begin{array}{c}\boldsymbol{E S} \\
\boldsymbol{R}\end{array}$ \\
\hline Sensor module & 2.09 & 4 & 4 \\
\hline $\begin{array}{c}\text { Computer module } \\
\text { Data acquisition instrument } \\
\text { module }\end{array}$ & 2.33 & 5 & 3 \\
\hline Send message module & 0.21 & 3 & 4 \\
\hline Physical transfer module & 0.18 & 5 & 3 \\
\hline Channel module & 2.19 & 3 & 4 \\
\hline Receive message module & 0.32 & 3 & 5 \\
\hline Database module & 1.61 & 4 & 4 \\
\hline Analysis tool module & 2.83 & 5 & 4 \\
\hline
\end{tabular}

Similarly, according to formula (4) (8), the fuzzy judgment matrix of each subsystem can be obtained in turn as follows:

1) Fuzzy judgment matrix of data acquisition system

$$
W=\left[\begin{array}{ccc}
0.5 & 0.75 & 1 \\
0.25 & 0.5 & 0.75 \\
0 & 0.25 & 0.5
\end{array}\right]
$$

2) Fuzzy judgment matrix of data transmission system

$$
W=\left[\begin{array}{cccc}
0.5 & 2 / 3 & 1 / 3 & 5 / 6 \\
1 / 3 & 0.5 & 1 / 6 & 2 / 3 \\
2 / 3 & 5 / 6 & 0.5 & 1 \\
1 / 6 & 1 / 3 & 0 & 0.5
\end{array}\right]
$$

3) Fuzzy judgment matrix of data processing system

$$
W=\left[\begin{array}{cc}
0.5 & 0 \\
1 & 0.5
\end{array}\right]
$$

According to formula (9) and above fuzzy judgment matrix, the weight coefficient of each function module is evaluated. Then, reliability of each subsystem which shows in table 6 is obtained on the basis of series RBD when system continuously works for 1000 hours. The failure rate of each subsystem is shown in table 7.

Table 6. Reliability of each subsystem.

\begin{tabular}{|c|c|}
\hline Subsystem name & Reliability \\
\hline Data acquisition system & 0.5466 \\
\hline Data transmission system & 0.1748 \\
\hline Data processing system & 0.7535 \\
\hline
\end{tabular}

\subsection{Reliability analysis of system}

$O P R$ and ESR of each function module are shown in table 5.

Table 7. Failure rate, $O P R$ and $E S R$ of each subsystem.

\begin{tabular}{|c|c|c|c|}
\hline Subsystem name & $\lambda\left(\mathbf{1 0}^{-\mathbf{3}}\right)$ & $\boldsymbol{O P} \boldsymbol{R}$ & $\boldsymbol{E S R}$ \\
\hline Data acquisition system & 0.604 & 5 & 4 \\
\hline $\begin{array}{c}\text { Data transmission } \\
\text { system }\end{array}$ & 1.744 & 5 & 5 \\
\hline Data processing system & 0.283 & 4 & 3 \\
\hline
\end{tabular}

Similarly, according to formula $(4) \sim(8)$, the fuzzy judgment matrix of system can be obtained in turn as follows:

$$
W=\left[\begin{array}{ccc}
0.5 & 0.25 & 0.75 \\
0.75 & 0.5 & 1 \\
0.25 & 0 & 0.5
\end{array}\right]
$$

According to formula (9) and above fuzzy judgment matrix, the weight coefficient of each subsystem is evaluated. Then reliability of system is 0.02407 on the basis of series RBD when system continuously works for 1000 hours. Failure rate and mean time before failure of the system are 0.003727 and 268 hours respectively.

The total failure rate and mean time before failure (MTBF) of the system are 0.0009785 and 1022 hours respectively by the traditional reliability method. Statistically, from 2013 to 2016, 36-time breakdowns happened in the cumulative working 11640 hours which means mean time before failure is actually 323 hours. It is obvious that the results evaluated by method in this paper are consistent with the actual situation. 


\section{Conclusion}

Industrial network system that is integrated with machine, electricity and control system coexist various failure modes in the long running process. It is necessary to evaluate and predict industrial network system reliability during development process in order to guarantee reliability. Therefore, a multi-layer reliability method were introduced based on RBD and AHP in this paper. In the proposed method the different influences of units on system reliability are considered. It is convenient to recognize the weak link and optimize the system reliability during design phase for engineers by using the proposed method. Through the case analysis, it shows that the method is more reasonable than the traditional reliability analysis method, and the reliability prediction result is consistent with the engineering practice.

\section{Reference}

1. X Wen, Y Shi, Z Niu. Electron. Prod. Relia. Environ.
Test, 3(2005):57-62

2. T Zhang, B Guo, Y Tan, et al. J. Syst. Eng. Electron, 27(2005):446-448, 470

3. $\mathrm{H} \mathrm{Yu}, \mathrm{X}$ Chen. J. Inst. Command. Tech. Equip, 13(2002):37-41

4. $\mathrm{X} \mathrm{Hu}, \mathrm{H}$ Zhang, $\mathrm{N}$ Lin, et al. Electron. Mech. Eng, 30(2014):22-26

5. X Qin, Z Chen. Comput. Eng, 31(2005):33-35

6. J Cao, C Wei. J. Syst. Simul, 22(2010):239-242

7. J Dou, W Chen, Z Zhong. J. Syst. Eng. Electron, 33(2011):954-957

8. $\mathrm{Z}$ Chen, $\mathrm{H}$ Qi, J Wei, et al. J. Eng. Des, 18(2011):407-411

9. J Wu, S Yan, L Xie. J. Tsinghua University, 52(2012): $15-20$

10. X Sun, W Li, Z Chen. J. Shanghai Jiaotong University, 45(2011):277-283

11. P Yan, A Che, $\mathrm{X}$ Tang. Comput. Integr. Manuf, 18(2012):332-336

12. Z Peng, W Sun, Fuzzy mathematics and its application - second edition (2007)

13. S Zeng, Reliability Design and Analysis(2011) 